\title{
GW23-e0774 PROFIBROTIC INFLUENCE OF HIGH GLUCOSE ON HUMAN CARDIAC FIBROBLAST FUNCTIONS: EFFECTS OF 606A AND IMIDAPRILAT
}

doi:10.1136/heartjnl-2012-302920a.124

Chi Jufang, Guo Hangyuan. Shaoxing People's Hospital

Objectives Recent studies have demonstrated an important role of chronic high glucose concentration for collagen deposition in fibroblasts. However, little is known about the action of angiotensin II type 1 receptor blocker and inflammatory cytokines on ACE inhibitor on matrix metalloproteinase (MMP) regulation and collagen synthesis in human cardiac fibroblasts. In this article, we determined the influence of chronic high glucose concentration on human cardiac fibroblasts functions and the effects of 606A and imidaprilat in these responses.

Methods Human cardiac fibroblasts were long-time exposure in normal or high glucose media in the absence or presence of $606 \mathrm{~A}$ or imidaprilat. We have determined their MMP-2 activities by using in-gel zymography. In addition, the collagen IV synthesis was evaluated by the means of ELISA. Results show that chronic high glucose concentration inhibits the activity of MMP-2 and accelerates collagen IV synthesis. When Equimolar mannitol was used as an osmotic control, the activity inhibition of MMP-2 were also observed, however, it is not as strong as that by using high glucose. Inhibition of MMP-2 activity and enhancement of collagen IV synthesis were reserved incompletely by 606A. But complete reservation of MMP-2 activity and collagen IV synthesis was observed by using imidaprilat in cultured media in the experiments.

Results show that chronic high glucose concentration inhibits the activity of MMP-2 and accelerates collagen IV synthesis. When Equimolar mannitol was used as an osmotic control, the activity inhibition of MMP-2 were also observed, however, it is not as strong as that by using high glucose. Inhibition of MMP-2 activity and enhancement of collagen IV synthesis were reserved incompletely by 606A. But complete reservation of MMP-2 activity and collagen IV synthesis was observed by using imidaprilat in cultured media in the experiments.

Conclusions Chronic high glucose inhibits the activity of MMP-2 and increases collagen IV synthesis by means of regulating MMP-2 mRNA expression in human cardiac fibroblasts through osmotic 


\section{ABSTRACTS}

and non-osmotic pathways. Inhibition of MMP-2 activity and enhancement of collagen IV synthesis were reserved incompletely by $606 \mathrm{~A}$. But complete reservation of MMP-2 activity and collagen IV synthesis was observed by using imidaprilat in cultured media in the experiments. 